\title{
Telemedicine application in patient with Diabetes, Hypertension and Rheumatoid arthritis $\llbracket A$ systematic review and meta-analysis
}

\author{
Yue Ma \\ Department of Nursing, Huashan hospital, Fudan University, Shanghai, China \\ Yan Zhao \\ Department of Nursing, Huashan hospital, Fudan University, Shanghai, China \\ Chongbo Zhao \\ Department of Neurology, Huashan hospital, Fudan University, Shanghai, China \\ Jiahong Lu \\ Department of Neurology, Huashan hospital, Fudan University, Shanghai, China \\ Hong Jiang \\ Department of Nursing, Huashan hospital, Fudan University, Shanghai, China \\ Yanpei Cao ( $\nabla$ yanpeicao@fudan.edu.cn) \\ Department of Nursing, Huashan hospital, Fudan University, Shanghai, China \\ Yafang Xu ( $\nabla$ yafang_xu@fudan.edu.cn) \\ Department of Nursing, Huashan hospital, Fudan University, Shanghai, China
}

\section{Systematic Review}

Keywords: Diabetes, hypertension, meta-analysis, rheumatoid arthritis, systematic review, telemedicine

Posted Date: June 4th, 2021

DOI: https://doi.org/10.21203/rs.3.rs-562285/v1

License: () (i) This work is licensed under a Creative Commons Attribution 4.0 International License. Read Full License 


\section{Abstract}

Background: Under the global epidemic condition $\nabla$ the telemedicine was widely used in the world, especially in the period of longterm care and treatment. The aim of study is to verify the effectiveness of telemedicine in the management of chronic disease by using scientific methods such as hypertension, diabetes, rheumatoid arthritis by using Meta-analysis and systematic review methods. The purpose of this study was to systematic review of the effect of telemedicine in chronic disease, so as to provide inspiration for chronic disease management in the future.

Methods: Article searching were performed using Web of Science, PubMed, MEDLINE, EMBASE and other library or database to retrieve articles which published from database and library establishment to October $31^{\text {st }}, 2020$. Literature quality assessment, systematic review and Meta-analysis were then performed.

Results: 12 articles were included in the literature quality assessment (7 diabetes,3 hypertensions, 2 rheumatoid arthritis), the article included in this study is of high quality. There are 7 articles included in the Meta-analysis, the result shows there have effect in glycosylated hemoglobin after 12 months of intervention ( $95 \% \mathrm{Cl}=-1.53,-0.16 ; \mathrm{Z}=2.42 ; \mathrm{P}=0.02)$, compare with 6 months $(95 \% \mathrm{Cl}=-1.32,-0.01 ; Z=1.99 ; P=0.05)$. It also showed that there was no significant difference in fasting blood glucose after 6 months of intervention $(95 \% \mathrm{Cl}=-1.19,0.21 ; Z=1.37 ; \mathrm{P}=0.17)$. Both systolic blood pressure $(95 \% \mathrm{Cl}=-12.79,-3.69 ; \mathrm{Z}=3.55 ; \mathrm{P}=$ $0.0004)$ and diastolic blood pressure $(95 \% \mathrm{Cl}=-9.90,-0.43 ; Z=2.14 ; P=0.03)$ showed statistically significant. Moreover, we also found positive influence of telemedicine about good behaviors and rehabilitation for rheumatoid arthritis patients.

Conclusion: The results showed that telemedicine had a positive effect on the management of diabetes, hypertension and rheumatoid arthritis, especially on the management of glycosylated hemoglobin and blood pressure. Telemedicine technologies have great promotion to access the medical services and improve the quality of care, especially for people with chronic diseases.

\section{Introduction}

During the global epidemic condition $\nabla$ the telemedicine was widely used in the world, especially in the period of long term care and treatment. Telemedicine have provided a feasible, credible technology(1), it has more advantages in chronic disease management, such as avoid the risk of the cross infection between patients, free from time and place constraints, wide range of applications faster and easier access to medical knowledge and enhanced communication between health-care workers(2). Therefore, Telemedicine as an important mode of health care has appeared on many occasions. Telemedicine is defined by the American Telemedicine Association (ATA) as the use of electronic communications tools to communicate medical information to a remote location for the purpose of improving a patient's health $(3,4)$. There were more intervention measures of interactive consultative and diagnostic services, such as online health education, telephone visit, remote guidance, telemonitoring, remote consultation, telehealth, web-based or internet-based consultation, mobile monitoring, mobile-health, promoting monitoring, mHealth, telecare $(5,6)$. At present, telemedicine can be composed of the following three parts: technology, functionality and applications. The components of the technological dimension can be grouped into three sets of variables: synchronicity, network design, and connectivity, information is transmitted and exchanged in these three ways. Consultation, diagnosis, mentoring and monitoring comprise the function of telemedicine. Advanced information exchange technologies have become the basis for telemedicine interventions, and functions can be applied in real life, such as treatment modality, site of care, disease entity and medical specialty, meanwhile, feedback is available when problems arise with telemedicine applications(6). Therefore, this is a good time for telemedicine to benefit chronic diseases. (Figure.1 Telemedicine model diagram)

With the development of global economy and the change of life style, chronic diseases have become an important public health problem affecting the 21st century(7). Chronic disease is defined as the diseases which have one or more of the following characteristics: they are permanent, leave residual disability, caused by nonreversible pathological alteration, require special training of the patient for rehabilitation, or may be expected to require a long period of supervision, observation, or care(8). The 
types of common chronic diseases mainly include the diabetes, chronic obstructive pulmonary disease, chronic bronchitis, emphysema, cardiovascular disease, mental disorder, mental illness and so on(9).

According to the WHO report, 68 per cent of the 56 million deaths worldwide are caused by chronic diseases, which have become the number one cause of human death $(10,11)$. Moreover, the increasing prevalence of chronic diseases has seriously affected people's lives, such as decline in the quality of life, impaired mobility, negative emotion, more complications, increase the economic burden and higher mortality(12).

Hypertension is one of the most important cause of premature death, one billion people worldwide suffer from the disease, and estimated 1.56 billion people will have hypertension by 2025(13). Statistically, over 180 million people have diabetes all over the world, may double in 2030, (14). Moreover, the incidence of rheumatoid arthritis in adults worldwide is $0.5 \%$, it has become one of the top 10 chronic diseases in China, and the incidence of rheumatoid arthritis in adults ranged from $0.5 \%$ to $1.0 \%$ in the United States $(4,10)$. They are common chronic diseases in the population.

In recent years, some studies on the intervention of chronic disease management through telemedicine have been carried out gradually, but there is no consensus about the effect of telemedicine which used in chronic disease management. the aim of this study focused on the effectiveness of the telemedicine intervention by systematic evaluation and meta-analysis.

\section{Methods}

\subsection{Selection of studies}

Inclusion and exclusion criteria of the literature are as follows. Inclusion criteria for participants:(1) used randomized-controlled trial or quasi-experimental designs (2) telemedicine was the main intervention in this investigation. (3) the disease type must be chronic. (4) the articles published in English and Chinese. Exclusion criteria for participants: participants cannot use related remote monitoring software tools.

\subsection{Search strategy}

The literature search was performed in the Cochrane, CINAHL, EBSCO, Medline, PubMed, EMBASE, Web of Science, JBI, NICE, Sinomed, CNKI (Chinese database), Weipu (Chinese database) and Wanfang (Chinese database) database. The articles searching time interval from database and library establishment to October $31^{\text {st }}, 2020$.

English searching terms used in the literature retrieval include: ("Telemedicine" OR "Remote Consultation" OR " Telehealth" OR "Telemonitoring " OR " Web-based" OR " mobile monitoring" OR "mobile health" OR" internet-based" OR " promoting monitoring "OR" mHealth" OR "Telecare")AND("Chronic Disease" OR "Arthritis" OR "Rheumatic Diseases" OR "Hypertension" OR "Digestive System Disease" OR "Coronary Disease" OR "Pulmonary Disease, Chronic Obstructive" OR "Dyslipidemias" OR "Kidney Diseases" OR "Diabetes Mellitus" OR "Asthma" OR "Liver Diseases" OR "Stroke" OR "Cognition Disorders" OR "Mental Disorders" OR "Neoplasms")AND(“Disease management" OR “Management"). Corresponding Chinese searching terms were used during the literature search in Chinese databases.

Full-text screening excluded literature:(1) Duplicate publication. (2) Inconsistent or poorly described interventions. (3) Nonrandomized controlled trials. (4) Random method error. (5) data required for the study were not provided. (6) inconsistent outcome measurements.

\subsection{Data extraction and critical appraisal}

Inclusion criteria and exclusion criteria were piloted by 2 researchers and disagreements were resolved by discussion with all authors. The two researchers screened the literature independently according to the inclusion criteria and exclusion criteria, and made a preliminary screening according to the title, keywords and abstract of the literature.

\subsection{Literature quality assessment}


The quality of each RCT was evaluated by the risk-of-bias assessment tool for RCT recommended by the Cochrane Handbook for Systematic Reviews of Interventions 5.0.2(15). Two researchers made the evaluations independently. A third researcher was consulted if there was any discrepancy. The quality of each QRCT was evaluated by the QRCT-assessment tool from the Joanna Briggs Institute, and the assessment was independently assessed by two researchers and. If they have inconsistent opinions after evaluating the quality of the literature, a third researcher will be invited to evaluate the quality of the literature again.

\subsection{Data retrieval}

The literature data were extracted into a detailed table, which included the following items: (1) Author, country, year of publication. (2) Age and gender. (3) Sample size of experimental group and control group. (4) Study disease categories. (5) Measures taken by the experimental group and the control group respectively. (6) Outcome indicators. (7) Duration of intervention. (8) Conclusion and discussion.

\subsection{Data synthesis and analysis}

Meta-analysis was performed using Revman 5.3 software according to inclusion and exclusion criteria for each study. We conducted a meta-analysis of three or more articles describing the same disease, which had the same outcome indicators and similar intervention duration. The final measured outcome measures are all continuous variables. We also calculated $95 \%$ confidence interval $(\mathrm{Cl})$. A fixed-effect model was applied if the heterogeneity from multiple studies was small (consistency test had a $p>0.05$ with $12 \leq 50 \%)$. Otherwise, a random-effects model was adopted if there was high heterogeneity $(p<0.05, I 2 \geq$ $50 \%)$.

\section{Results}

\subsection{Study selection and characteristics}

The figure below shows the chart of the article selection process. The search resulted in 3585 articles, 2756 articles did not address after the title and abstract were assessed. 501 articles full text were read. 489 articles were excluded in the final stage assessment. 12 articles were included in the study. (Figure.2 Literature screening flow chart)

\subsection{Results of literature retrieval}

The quality evaluation of 12 articles included in the literature(16-27) (Table 1 Characteristics of included studies). It was published between 2006 and 2020. There were seven references included in the final meta-analysis, four articles focused on diabetes $(16,19,20,23)$, and three articles studies hypertensions(25-27)

\subsection{Risk of bias assessment}

Baseline characteristics were reported in all the articles included in the literature quality assessment, there were eight literatures $(17,18,20,22-24,26,27)$ with low bias risk by random method, eight articles $(17,18,20,22-24,26,27)$ have achieved the generation of standard random sequence and proper allocation concealment, only one article of allocation concealment is classified as having a high risk of bias(25). Four of them used blinded methods for subjects, trainers, and evaluators $(16,17,21$, 24), the two articles clearly mentioned that the study subjects, interveners and outcome measurers were not blinded due to intervention methods $(22,26)$. Most of the literature reports the missing data and the reasons except for one article(19) (Figure.3 Chart of quality risk assessment of related literature) All of the literature included in this study is considered high quality after assessment.

\subsection{Meta-analysis}

\subsubsection{Outcome measures}

There are 4 articles $(16,19,20,23)$ used glycosylated hemoglobin and fasting blood glucose as the primary outcome. High fasting blood glucose concentration (FBG) has been considered as one of the potential risk factors for small arterial stiffness, it 
is tend to cause diabetic complications(28). glycosylated hemoglobin, an indirect measure of mean blood glucose index, it could reflect the level of blood glucose over the previous 2-3 months(29). Meta-analysis of glycosylated hemoglobin at 6 months $(19,20,23)$ and 12 months $(16,20,23)$ was performed in the four literatures according to the different intervention duration.

The type of disease studied in the other three literatures is hypertension(25-27), and telemedicine is the main intervention method. The fluctuation of blood pressure become a major risk factor for stroke and heart disease in the population(30), all of these three articles used systolic blood pressure and diastolic blood pressure as primary outcome, so we conducted a metaanalysis to test the effect of intervention.

\subsubsection{Meta-analysis of diabetes}

Jun Yang Lee(20), Han Yun(23)and Feng ya kun et al(19)reported the experimental data of the intervention for 6 months in the literature, we found no improvement in glycosylated hemoglobin and fasting blood glucose of three RCTs. However, according to the other studies reported that the significant difference in glycosylated hemoglobin after intervention of 12 month(16, 20, 23). Meta-analysis was conducted at two time points of 6 months respectively according to the duration of intervention.

\section{Meta-analysis of glycosylated hemoglobin after 6 months of intervention}

Because of the high statistical heterogeneity $\left(l^{2}=94 \%\right)$, the random effect model was used. The results showed that there was no significant difference in glycosylated hemoglobin between the intervention group and the control group after 6 months of intervention. ( $M D=-0.66 ; 95 \% \mathrm{Cl}=-1.32,-0.01 ; Z=1.99 ; P=0.05)$ (Figure. 4 Forest plots of glycosylated hemoglobin after 6 months of intervention between the intervention group and the control group)

\section{Meta-analysis of fasting blood glucose after 6 months of intervention}

This set of data uses a random effects model due to its high statistical heterogeneity $\left(I^{2}=78 \%\right)$. The results showed that there was no significant difference in fasting blood glucose between the experimental group and the control group $(\mathrm{MD}=-0.49 ; 95 \% \mathrm{Cl}=-1.19,0.21 ; \mathrm{Z}=1.37 ; \mathrm{P}=0.17$ ) (Figure. 5 Forest plots of fasting blood glucose after 6 months of intervention between the intervention group and the control group)

\section{Meta-analysis of glycosylated hemoglobin after 12 months of intervention}

A random-effect model was adopted since there was high statistical heterogeneity $\left(I^{2}=99 \%\right)$. However, analysis of three other 12-month intervention duration studies $(16,20,23)$ showed statistically significant differences in glycosylated hemoglobin between the intervention and control groups (MD=-0.84;95\%Cl=-1.53, $-0.16 ; Z=2.42 ; P=0.02$ ) (Figure. 6 Forest plots of glycosylated hemoglobin after 12 months of intervention between the intervention group and the control group)

\subsubsection{Meta-analysis of hypertension}

This meta-analysis included three articles studied on hypertension(25-27). The results showed that under the intervention of telemedicine measures, the difference between the experimental group and the control group in systolic blood pressure and diastolic blood pressure was statistically significant after 6 months of intervention, and the difference in systolic blood pressure was remarkable.

\section{Meta-analysis of systolic blood pressure after 6 months of intervention}

The systolic blood pressure in the experimental group and the control group after 6 months of intervention in the included literatures were analyzed. A random-effects model was used because there was high statistical heterogeneity (SBP: $\left.1^{2}=91 \%\right)$. The results showed that there were statistically significant differences in systolic blood pressure between the group receiving

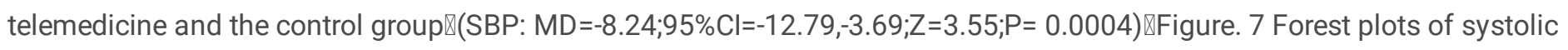

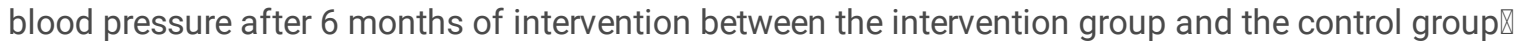




\section{Meta-analysis of diastolic blood pressure after 6 months of intervention}

Three articles(25-27) report the diastolic blood pressure at 6 months of intervention. The random effect model was used because of the high statistical heterogeneity (DBP: 12=98\%). The results showed that there were statistically significant differences in diastolic blood pressure between the intervention group the control group $\mathbb{( D B P}: \mathrm{MD}=-5.16 ; 95 \% \mathrm{Cl}=-9.90,-0.43$; $Z=2.14 ; P=0.03) \otimes F i g u r e .8$ Forest plots of diastolic blood pressure after 6 months of intervention between the intervention group

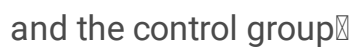

\section{Discussion}

\subsection{Telemedicine was less effective in reducing fasting blood glucose in patients with diabetes}

From the result of meta-analysis, there was no statistically significant difference of fasting blood glucose levels between the experimental group and the control group. Angeles 's study(31)also showed that compared with the usual group, the metaanalysis of fasting blood glucose after intervention of 3 months, 6 months and 12 months was not statistically significant. In a study of type 2 diabetes(20), 120 people were included in the intervention group, it was found that the difference between the intervention group and the control group was not statistically significant. However, Han Yun et al(23) figure out that significant differences in fasting blood glucose levels at the 3th ,6th and 12th months after intervention, and the fasting blood glucose levels in the intervention group were significantly lower than those in the control group. Perhaps, the reason for the difference may be related to the duration of diabetes and the severity of the disease in participants of the intervention group. (31).

\subsection{Duration effect of telemedicine on glycosylated hemoglobin in patients with diabetes}

Our result shows there was significant difference in glycosylated hemoglobin after intervention of 12 months, but there was no significant difference in glycosylated hemoglobin between the intervention group and the control group after 6 months. Therefore, we supposed that changes in glycosylated hemoglobin may have time effects.

Our results are similar to Viana et al(32)凶they performed a meta-analysis of glycosylated hemoglobin with telecare intervention, a total of 1,782 people were included in the study, the length of most studies was 6 months, and they also found no significant improvement in glycosylated hemoglobin. Moreover, we also find the long-term duration of intervention has positive effects on the control of glycosylated hemoglobin, the consequence is similar to the meta-analysis of Lee and Timpel et al. Lee et al figure out that telemedicine system can be positive in type 1 diabetes patients after intervention (33), the telemedicine system used in most trials were relatively simple and involved data transmission of blood glucose data with feedback, it turned out that the studies lasted at least 6 months in duration or longer, and that the effect of telemedicine was more obvious. Furthermore, Timpel et al(34) pointed out the great benefit in glycosylated hemoglobin reduction after telemedicine intervention, especially the communication and interactive functions of telemedicine, they find that digital health education was statistically significant in the long-term intervention duration of 12 months. Some of the original RCTs also reflects the time effects. Wakefield et al(35) conducted original research, the intervention group included home remote monitoring and nursing management over 6 months, the result shows that the difference between the intervention group and the control group was significant at 6 months, but if the intervention was withdrawn 6 months after the intervention, the glycosylated hemoglobin indicators of the intervention group and the control group will gradually tend to be consistent. Similar results also reflected in the study of Stelios Fountoulakis et al(17), telemonitoring which combined with a management and feedback system based on transmitted data has been proven effective in rapidly reducing glycosylated hemoglobin, the results showed significant reductions in glycosylated hemoglobin at both 3 months and 6 months compared with baseline, and the slightly attenuated at 6 months after its discontinuation. In the five-year follow-up study by shea et al(21), a total of 1665 people were recruited, the telemedicine group regularly implemented home unit management, nurse case management, and remote consultation activities, follow-up examinations were conducted at 1-year intervals, finally, the intervention group had net improvement in glycosylated hemoglobin compared with usual group.

In conclusion, most studies have shown that telemedicine has a positive effect on the management of diabetes, but the best time to intervene is different, from our study we found that telemedicine has a positive effect at 12 months. Telemedicine is a 
process of long-term intervention and will be more effective over time, this improvement could be attributed to better compliance due to a more frequent contact by using telemedicine(17).

\subsection{Blood pressure can be improved by telemedicine intervention}

The results of our meta-analysis showed that there was significant difference in systolic blood pressure and diastolic blood pressure after intervention of 6 months. Many studies have found that blood pressure can be improved by telemedicine intervention, although the duration of the intervention in many studies was different.

Our results of the study are similar to Verberk et al(36), it was a meta-analysis included nine randomized clinical trials, their research showed that the systolic blood pressure and diastolic blood pressure of the intervention group are significantly decreased by using telecare, this measure not only controls blood pressure effectively, but also enables patients to adhere to treatment. Another system review figure out that home remote monitoring does a better job of controlling blood pressure than other approaches, they selected some articles that examined populations of patients with hypertension, it is worth noting that in most cases the studies found a significant drop in blood pressure in the first 3 months of remote monitoring(37). However, an RCT study(38)which involved 387 participants, the interventions were instructed by trained nurses, such as blood pressure monitoring, answer questions, link device to the participant's home, both systolic blood pressure and diastolic blood pressure were found to have significance after 12 months of intervention.

Most articles in RCTs have confirmed the positive effect of telemedicine on blood pressure, it can help support doctors and nurse for closer, continuous follow-up of patients with hypertension, although the duration of the intervention was different. (3941)

\subsection{Integration of telemedicine studies in patients with rheumatoid arthritis}

Rheumatoid arthritis is one of the most prevalent chronic inflammatory diseases. The main symptoms of rheumatoid arthritis include rheumatoid nodules, pulmonary involvement or vasculitis, and systemic comorbidities(42). Patients with rheumatoid arthritis have a longer disease duration, it can cause joint deterioration and functional disability, eventually leading to unfavorable disease outcomes and seriously affected the activity of daily life(43). Therefore, the long-term management of rheumatoid arthritis patients with telemedicine as the main intervention method is developed gradually.

The main intervention in Ferwerda 's research(18) is a cognitive behavioral methods to manage the patients with rheumatoid arthritis. The results showed the effectiveness of Internet-based tailored cognitive behavioral interventions for rheumatoid arthritis patients with psychological risks. Yuqing Song(22) conducted a RCT study to observe the impact of telemedicine education on drug compliance and disease activity of rheumatoid arthritis patients, the results showed that it has not improved symptoms of patients by using remote medical education, but it can enhance the medication compliance of patients. Both articles $(18,22)$ showed that telemedicine could promote positive behaviors in patients with rheumatoid arthritis, the positive behaviors influence of telemedicine is benefit for rheumatoid arthritis patients, Optimistically, By searching the relevant literature, telemedicine will have the opportunity to become an important tool in the management of patients with rheumatoid arthritis, at the same time, there are some uncertainties in the application of the Internet for rheumatoid arthritis patients.(44), so we still need more RCT study to prove the effect of telemedicine on rheumatoid arthritis, which will be a key point for the future research.

\subsection{The advantages and disadvantages of telemedicine}

Nowadays, the technology of telemedicine is frequency to be used. Telemedicine technology has also been applied to various fields of medicine, and some diseases can be treated and cared at home (1). telemedicine can provide long-term care and treatment for people with chronic diseases through the website or other telecommunication equipment, which is helpful to reduce the risk of virus spread in period of COVID-19 global pandemic. Many articles have confirmed telehealth and telemedicine technologies have the potential to increase access to healthcare services and make full use of medical resource, it could also help to change the treatment plan in time and improve the service quality.(45). Telemedicine has also been be 
considered as a cost-effective methods for the management of chronic diseases(46). However, there still has a big barrier for the patient who has difficulty and anxiety to use computer or mobile especially for elderly people.(47).

\section{Conclusions And Recommendation}

This study conducted a meta-analysis and systematic review to verify the effect of telemedicine application on patients with diabetes, hypertension and rheumatoid arthritis. The results indicated that telemedicine had a positive effect on the management of diabetes, hypertension and rheumatoid arthritis, Telemedicine technologies have great promotion to access the medical services and improve the quality of care, especially for people with chronic diseases (48) Although telemedicine also has some vulnerabilities such as data leakage and other network security issues, it can break through the distance between time and space and reach the insufficient medical resource area, this is the benefit of telemedicine (5).

\section{Declarations}

Competing interests: The authors declare no competing interests.

\section{Author Contributions}

Yue Ma assess the quality evaluation of literature and Writing - Original draft preparation; Yan Zhao Assess the quality evaluation of literature; Chongbo Zhao, Jiahong Lu and Hong Jiang review and revise the manuscript ; Yanpei Cao and Yafang Xu designed study and revise the manuscript.

\section{Availability of data and materials}

All data generated or analysed during this study are included in this published article [and its supplementary information files].

\section{Funding}

The work described in this paper was supported in full by a grant from the Fu Xing Nursing Scientific Research Fund of Fudan University, Shanghai, China [grant number FNF202063].

\section{Declaration of interest}

None.

\section{Ethical approval and consent to participate}

Not applicable.

\section{Acknowledgments}

The authors thank all the participants in the study.

\section{References}

1. Sood S, Mbarika V, Jugoo S, Dookhy R, Doarn CR, Prakash N, et al. What Is Telemedicine? A Collection of 104 PeerReviewed Perspectives and Theoretical Underpinnings. Telemedicine and e-Health. 2007;13(5):573-90.

2. Craig J, Patterson V. Introduction to the practice of telemedicine. J Telemed Telecare. 2005;11(1):3-9.

3. American Telemedicine Association.Telemedicine glossary[EB/OL]. [2019-08-30].http://thesource.americ antelemed.org/resources/telemedicine-glossary.

4. LU Jun ZQ. Application progress of telemedicine technology in home $₫$ based rehabilitation of joint function among patients with rheumatoid arthritis. CHINESE NURSING RESEARCH. 2020. 
5. Burke BL, Jr., Hall RW. Telemedicine: Pediatric Applications. Pediatrics. 2015;136(1):e293-308.

6. Bashshur R, Shannon G, Krupinski E, Grigsby J. The taxonomy of telemedicine. Telemed J E Health. 2011;17(6):484-94.

7. Zongxia ZGYWYGC. Enlightenment of foreign management model of chronic diseases to chronic care management in China. Chin J Mod Nurs. 2017;Vol.23『No.6.

8. Chatfield M. Dictionary of Health Services Management, 2d ed. Health Care Management Review1983.

9. Yu-di GYYT-tY. Analysis of main risk factors and countermeasures of chronic diseases based on systems archetypes. Chinese Journal of General Practice. 2019.

10. Dr Margaret Chan DOC. Global status report on NCDs 2014. World Health Organization. 2014.

11. Zongxia ZGYWYGC. Enlightenment of foreign management model of chronic diseases to chronic care management in China. Chinese Journal of Modern Nursing. 2017.

12. Mohammed MA, Stevens A. The value of administrative databases. BMJ. 2007;334(7602):1014-5.

13. Yang T, Zubcevic J. Gut-Brain Axis in Regulation of Blood Pressure. Front Physiol. 2017;8:845.

14. Wild S, Roglic G, Green A, Sicree R, King H. Global Prevalence of Diabetes: Estimates for the year 2000 and projections for 2030. Diabetes Care. 2004;27(5):1047-53.

15. Higgins, JPT. Cochrane handbook for systematic reviews of interventions version 5.0. 2: Wiley-Blackwell.

16. Shea S, Weinstock RS, Starren J, Teresi J, Plamas W, Field L, et al. A randomized trial comparing telemedicine case management with usual care in older, ethnically diverse, medically underserved patients with diabetes mellitus. Journal of the American Medical Informatics Association. 2006;13(1):40-51.

17. Stelios Fountoulakis, Labrini Papanastasiou, Alexandros Gryparis, Athina Markou, Piaditis G. Impact and duration effect of telemonitoring on $\mathrm{HbA1c}, \mathrm{BMI}$ and cost in insulin-treated diabetes mellitus patients with inadequate glycemic control $\mathrm{A}$ randomized controlled study. HORMONES. 2015.

18. Ferwerda M, van Beugen S, van Middendorp H, Spillekom-van Koulil S, Donders ART, Visser H, et al. A tailored-guided internet-based cognitive-behavioral intervention for patients with rheumatoid arthritis as an adjunct to standard rheumatological care. Pain. 2017;158(5):868-78.

19. Ya-kun $\otimes$ F, Xiao-kun LY-jG. The application of telemedicine in the integrated management of type 2 diabetes mellitus. Chinese Journal of Diabetes. 2016.

20. Lee JY, Chan CKY, Chua SS, Ng CJ, Paraidathathu T, Lee KKC, et al. Telemonitoring and Team-Based Management of Glycemic Control on People with Type 2 Diabetes: a Cluster-Randomized Controlled Trial. J Gen Intern Med. 2019;35(1):8794.

21. Shea S, Weinstock RS, Teresi JA, Palmas W, Starren J, Cimino JJ, et al. A Randomized Trial Comparing Telemedicine Case Management with Usual Care in Older, Ethnically Diverse, Medically Underserved Patients with Diabetes Mellitus: 5 Year Results of the IDEATel Study. Journal of the American Medical Informatics Association. 2009;16(4):446-56.

22. Song YQ, Reifsnider E, Zhao SP, Xie X, Chen H. A randomized controlled trial of the Effects of a telehealth educational intervention on medication adherence and disease activity in rheumatoid arthritis patients. Journal of Advanced Nursing. 2020;76(5):1172-81.

23. HAN Yun XY, YE Xillhlla, LI Xiaona, YANG Ping $\ W U$ Yan, CHEN Liye, WU Haili. Application of chronic disease management model with Internet plus in patients with type 2 diabetes melli tus. Chinese Journal of Nursing. 2018.

24. Sood A, Watts SA, Johnson JK, Hirth S, Aron DC. Telemedicine consultation for patients with diabetes mellitus: a cluster randomised controlled trial. Journal of Telemedicine and Telecare. 2018;24(6):385-91.

25. Margolis KL, Asche SE, Dehmer SP, Bergdall AR, Green BB, Sperl-Hillen JM, et al. Long-term Outcomes of the Effects of Home Blood Pressure Telemonitoring and Pharmacist Management on Blood Pressure Among Adults With Uncontrolled Hypertension. JAMA Network Open. 2018;1(5).

26. McManus R, Mant J, Bray EP, Holder R, Jones MI, Greenfield S, et al. Telemonitoring and self-management in the control of hypertension (TASMINH2): a randomised controlled trial. Lancet. 2010;376(9736):163-72. 
27. Jia L. Application of chronic disease management model of hospital community integration based on"internet+"for elderly patients with hypertension. Chinese Nursing Research. 2019.

28. Zheng M, Zhang X, Chen S, Song Y, Zhao Q, Gao X, et al. Arterial Stiffness Preceding Diabetes. Circulation Research. 2020;127(12):1491-8.

29. Nomura K, Inoue K, Akimoto K. A two-step screening, measurement of HbA1c in association with FPG, may be useful in predicting diabetes. PLoS One. 2012;7(4):e36309.

30. Slark J, Khan MS, Bentley P, Sharma P. Knowledge of blood pressure in a UK general public population. Journal of Human Hypertension. 2014;28(8):500-3.

31. Angeles RN, Howard MI, Dolovich L. The Effectiveness of Web-Based Tools for Improving Blood Glucose Control in Patients with Diabetes Mellitus: A Meta-Analysis. Canadian Journal of Diabetes. 2011;35(4):344-52.

32. Viana LV, Gomes MB, Zajdenverg L, Pavin EJ, Azevedo MJ, Brazilian Type 1 Diabetes Study G. Interventions to improve patients' compliance with therapies aimed at lowering glycated hemoglobin ( $\mathrm{HbA1c}$ ) in type 1 diabetes: systematic review and meta-analyses of randomized controlled clinical trials of psychological, telecare, and educational interventions. Trials. 2016;17:94.

33. Lee SWH, Ooi L, Lai YK. Telemedicine for the Management of Glycemic Control and Clinical Outcomes of Type 1 Diabetes Mellitus: A Systematic Review and Meta-Analysis of Randomized Controlled Studies. Frontiers in Pharmacology. 2017;8.

34. Timpel P, Oswald S, Schwarz PEH, Harst L. Mapping the Evidence on the Effectiveness of Telemedicine Interventions in Diabetes, Dyslipidemia, and Hypertension: An Umbrella Review of Systematic Reviews and Meta-Analyses. Journal of Medical Internet Research. 2020;22(3).

35. Wakefield BJ, Holman JE, Ray A, Scherubel M, Adams MR, Hills SL, et al. Outcomes of a Home Telehealth Intervention for Patients with Diabetes and Hypertension. Telemedicine and e-Health. 2012;18(8):575-9.

36. Verberk WJ, Kessels AGH, Thien T. Telecare is a valuable tool for hypertension management, a systematic review and metaanalysis. Blood Pressure Monitoring. 2011;16(3):149-55.

37. Paré G, Moqadem K, Pineau G, St-Hilaire C. Clinical Effects of Home Telemonitoring in the Context of Diabetes, Asthma, Heart Failure and Hypertension: A Systematic Review. Journal of Medical Internet Research. 2010;12(2):e21.

38. Artinian NT, Flack JM, Nordstrom CK, Hockman EM, Washington OGM, Jen K-LC, et al. Effects of Nurse-Managed Telemonitoring on Blood Pressure at 12-Month Follow-Up Among Urban African Americans. Nursing Research. 2007;56(5):312-22.

39. Ferrari R. The Role of Telemedicine in Hypertension Management: Focus on Blood Pressure Telemonitoring. Current hypertension reports. 2015;17:535.

40. Rogers M, Buchan D, Small D, Stewart C, Krenzer B. Telemedicine improves diagnosis of essential hypertension compared with usual care. Journal of telemedicine and telecare. 2002;8:344-9.

41. Omboni S, Ferrari R. The Role of Telemedicine in Hypertension Management: Focus on Blood Pressure Telemonitoring. Current Hypertension Reports. 2015;17(4).

42. Smolen JS, Aletaha D, Mclnnes IB. Rheumatoid arthritis. The Lancet. 2016;388(10055):2023-38.

43. Malm K, Bremander A, Arvidsson B, Andersson ML, Bergman S, Larsson I. The influence of lifestyle habits on quality of life in patients with established rheumatoid arthritis-A constant balancing between ideality and reality. Int J Qual Stud Health Well-being. 2016;11:30534.

44. Piga M, Cangemi I, Mathieu A, Cauli A. Telemedicine for patients with rheumatic diseases: Systematic review and proposal for research agenda. Seminars in Arthritis and Rheumatism. 2017;47(1):121-8.

45. Lamothe L, Fortin JP, Labbé F, Gagnon MP, Messikh D. Impacts of telehomecare on patients, providers, and organizations. Telemed J E Health. 2006;12(3):363-9.

46. Gaikwad R, Warren J. The role of home-based information and communications technology interventions in chronic disease management: a systematic literature review. Health Informatics J. 2009;15(2):122-46. 
47. Lamothe L, Fortin J-P, Labbé F, Gagnon M-P, Messikh D. Impacts of Telehomecare on Patients, Providers, and Organizations. Telemedicine journal and e-health : the official journal of the American Telemedicine Association. 2006;12:363-9.

48. McLean S, Sheikh A, Cresswell K, Nurmatov U, Mukherjee M, Hemmi A, et al. The Impact of Telehealthcare on the Quality and Safety of Care: A Systematic Overview. PloS one. 2013;8:e71238.

\section{Tables}

Table 1. Characteristics of included studies $(n=12)$ 


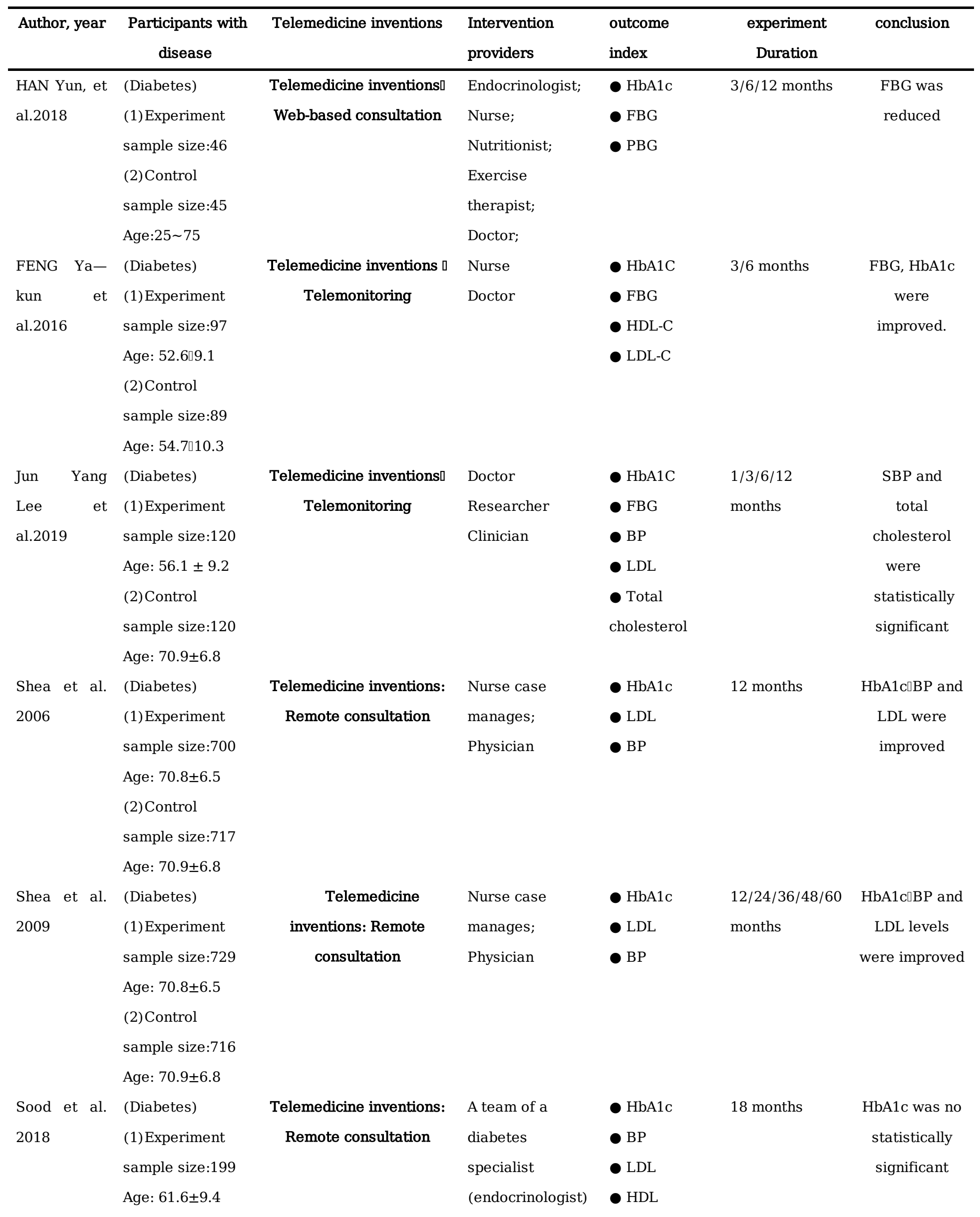


(2) Control

sample size:83

Age: $61.1 \pm 10.0$

Fountoulakis (Diabetes)

et al. 2015

(1) Experiment

sample size:70

Age: $55.2 \pm 16.1$

(2) Control

sample size:35

Age: $55.4 \pm 19.2$

LI Jia et al. (Hypertension) 2019

(1) Experiment

sample size:81

Age: $70.2 \pm 6.5$

(2) Control

sample size:81

Age: $69.2 \pm 6.7$

Margolis et (Hypertension)

al. 2018

(1) Experiment

sample size:228

Age: $62.0 \pm 11.7$

(2) Control

sample size:222

Age: $60.2 \pm 12.2$

McManus et (Hypertension)

al. 2010

(1) Experiment

sample size:234

Age: $66.6 \pm 8.8$

(2) Control

sample size:246

Age: $66.2 \pm 8.8$

Song et (Rheumatoid

al.2020

arthritis)

(1) Experiment

sample size:41

(2) Control

sample size:36

Age: $55.26 \pm 10.84$

Ferwerda et (Rheumatoid

al. 2017

Arthritis)

(1) Experiment

sample size:62 and a nurse

practitioner

Telemonitoring

Outpatient

$\mathrm{HbA1c}$

$3 / 6 / 6$

months

telemonitoring

Department

BMI

after

effective in

Endocrinologists

Cost

discontinuation.

rapidly

Doctor

reducing

HbA1c.

Telemedicine inventions: Nurse

BP

6months

BP was

Internet-based Doctor

consultation:

\section{Telemedicine inventions: Pharmacists \\ Telemonitoring \\ Doctor \\ $\mathrm{BP}$ \\ $6 / 12 / 18 / 54$ \\ months}

BP was

decreased

after 12

months

Telemedicine inventions: Research team; • BP 6/12months Telemonitoring Family doctor.

Telemedicine

Nurses

Medication

3/6 months

inventions: Telemonitoring compliance

Disease activity

Beck

Depression

3/6/9/12

Internet-based

consultation
Inventory
The

BP changed significantly after 6 months and 12 months

intervention group had significantly higher medication adherence patients with psychological distress have reduced 
(2) Control

sample size:71

Age: $56.35 \pm 10.00$
- Negative

$\operatorname{mood}$

- Anxiety

Satisfaction

(HbA1c: glycosylated hemoglobin; FBG: Fasting Blood Glucose; PBG: Postprandial Blood Glucose; FPG: Fasting Plasma Glucose; HDL-C: high-density lipoprotein cholesterol; LDL-C: low-density lipoprotein cholesterol; BMI: Body Mass Index; BP: Blood Pressure; LDL: Low Density Lipoprotein; HbA1c: glycosylated hemoglobin; HDL: high density lipoprotein; SBP: Systolic Blood Pressure; DBP: Diastolic Blood Pressure[

\section{Figures}

- Internet education

- telephone visit

- remote guidance

- telemonitoring

- remote consultation

- telehealth

- web-based consultation

- mobile monitoring

- mobile-health(mhealth)

- internet-based

consultation

- promoting monitoring

- telecare..

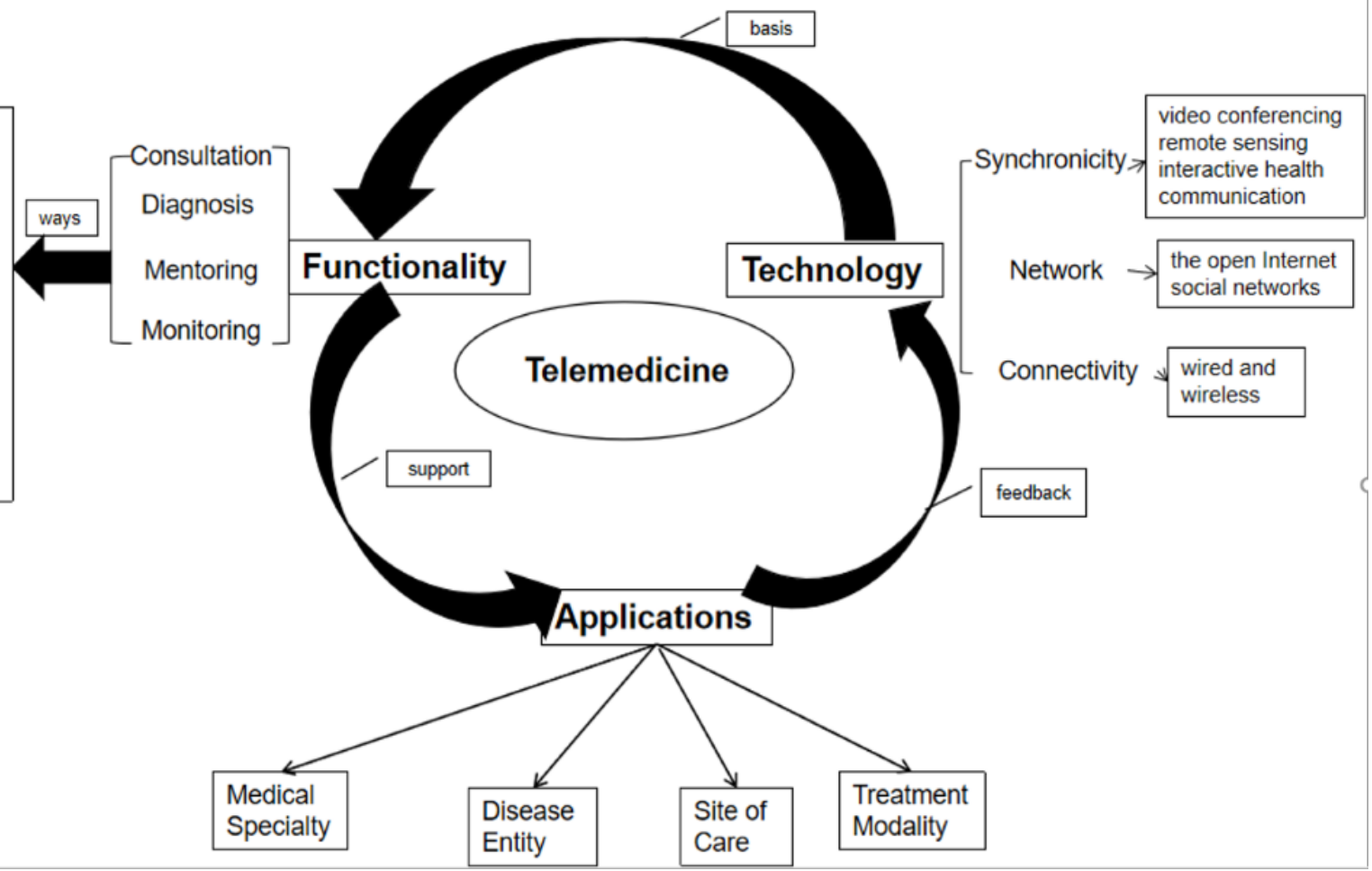

\section{Figure 1}

Telemedicine model diagram 


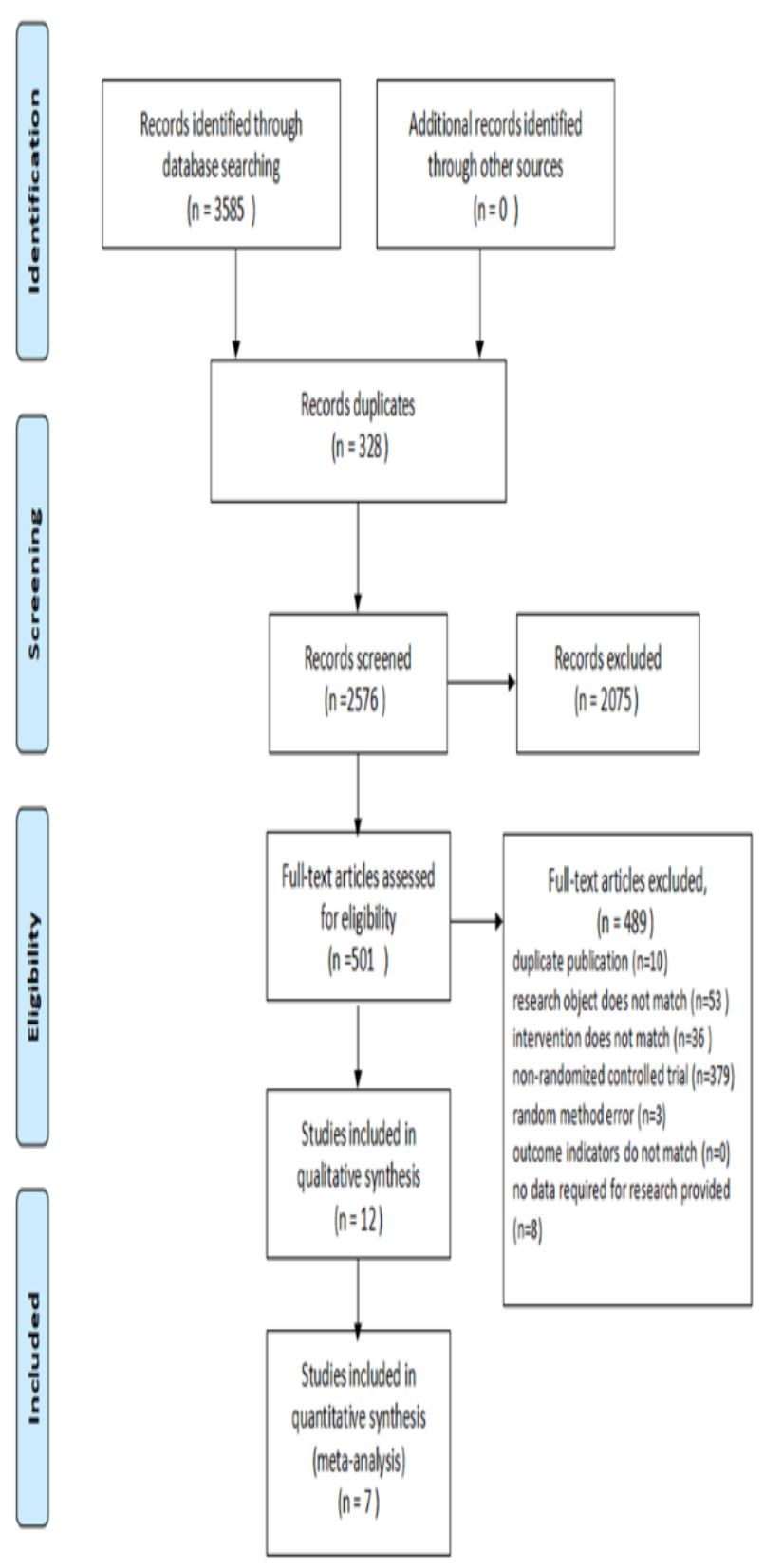

Figure 2

Literature screening flow chart 
Random sequence generation (selection bias)

Allocation concealment (selection bias)

Blinding of participants and personnel (performance bias)

Blinding of outcome assessment (detection bias)

Incomplete outcome data (attrition bias)

Selective reporting (reporting bias)

Other bias
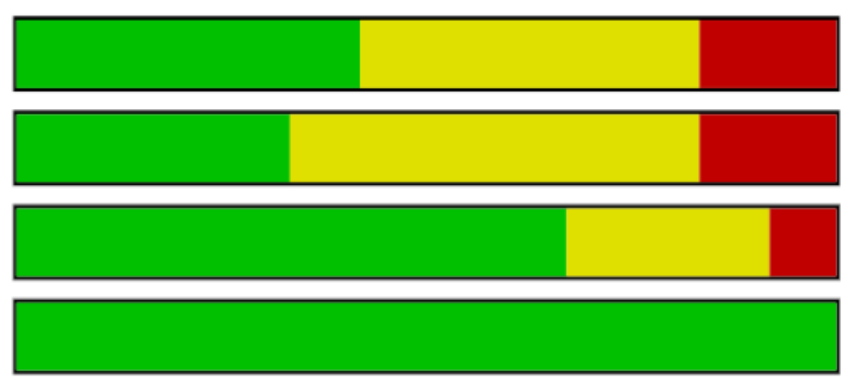

\section{Other bias}

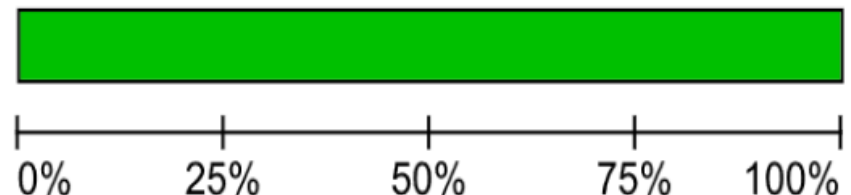

Low risk of bias

\section{Figure 3}

Chart of quality risk assessment of related literatur

\section{Experimental Control Mean Difiterence}

Mean Difference

Study or Subgroup Mean SD Total Mean SD Total Weight N. Random, 55\% Cl

IV.Random,95\% Cl

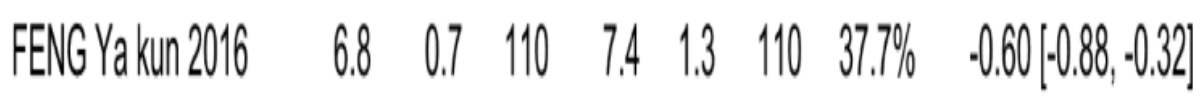

HANYUn2018

8.022 .00

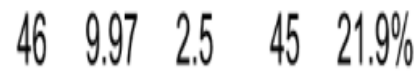

$-1.95[-2.90,-1 \cdot 00]$

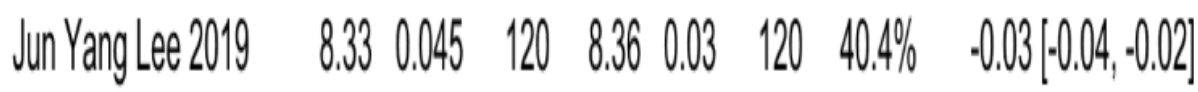

Total $195 \%$ C Cl)

$276 \quad 275 \quad 100.0 \%$

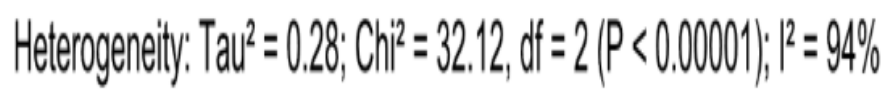

Testforovereal flect: $:=1.99(P=0.05)$

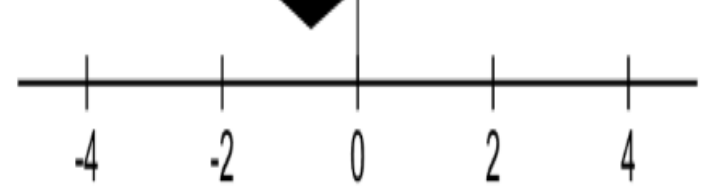

Favours [experimential) Favours [control]

Figure 4

Forest plots of glycosylated hemoglobin after 6 months of intervention between the intervention group and the control group 


\section{Experimental Control Mean Difference Mean Difference}

\section{Studvor Subgroup Mean SD Total Mean SD Total Weight IV.Random, 95\% Cl IV.Random, 95\% Cl}

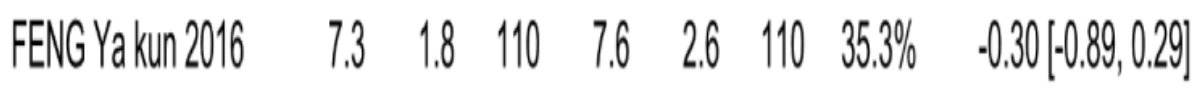

HANYun2018 $\quad 8.31 \quad 2.98 \quad 46 \quad 10.33 \quad 3.43 \quad 45 \quad 17.6 \%$

Jun Yang Lee $2019 \quad 8.760 .195 \quad 120 \quad 8.82 \quad 0.175 \quad 120 \quad 47.1 \%$

Total $(95 \%$ C C $) \quad 276 \quad 275 \quad 100,0 \% \quad \cdot 0.49[\cdot 1.19,0.21]$

Heterogeneity: $T^{2}{ }^{2}=0.27 ; \mathrm{Ch}^{2}=9.06$, of $=2(P=0.01) ; 1^{2}=78 \%$

Test for overall effect: $z=1,37(P=0.17)$

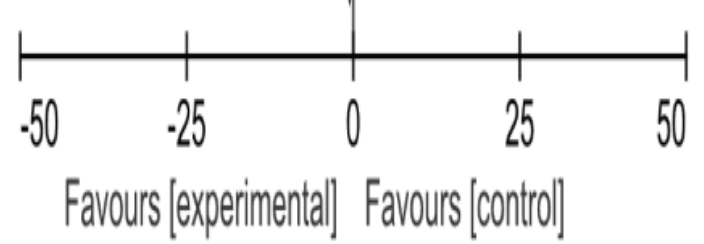

Figure 5

Forest plots of fasting blood glucose after 6 months of intervention between the intervention group and the control group Experimental Control Mean Difference Mean Difference Study or Subgrourp Mean SD Total Mean SD Total Weight IV, Random, 95\% C Cl N, Random, 95\% Cl HAN Yun 2018 $\quad 7.27 \quad 1.87 \quad 46 \quad 9.532 .02 \quad 45 \quad 24.9 \% \quad-2.26[-3.06,-1.46]$ Jun Yang Lee 2019 $\quad 8.690 .055 \quad 120 \quad 8.70 .04 \quad 120 \quad 37.8 \% \quad-0.01[[-0.02,0.00]$ $\begin{array}{lllllllll}\text { Shea 2006 } & 6.97 & 1.12 & 700 & 7.71 & 1.4 & 717 & 37.3 \% & -0.74[[-0.87,-0.61]\end{array}$

Total $(95 \%$ C C $) \quad 866 \quad 882 \quad 100.0 \% \quad \cdot 0.84[-1.53,-0.16]$

Heterogeneity: Tau $^{2}=0.32 ; \mathrm{Ch}^{2}=146.95$, af $=2(P<0.00001) \mathrm{r}^{2}=99 \%$

Test for overall effect: $z=2.42(P=0.02)$

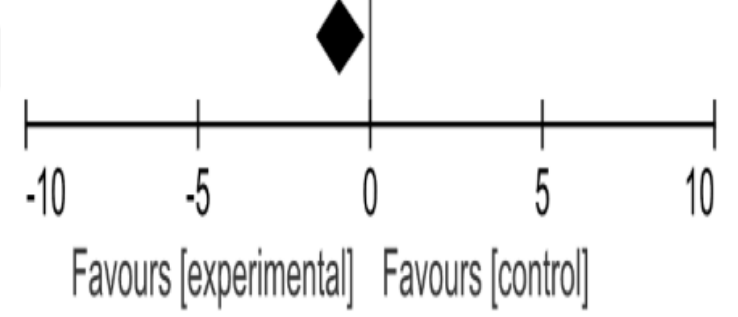

Figure 6

Forest plots of glycosylated hemoglobin after 12 months of intervention between the intervention group and the control group 
Experimental Control Mean Difference Mean Difference

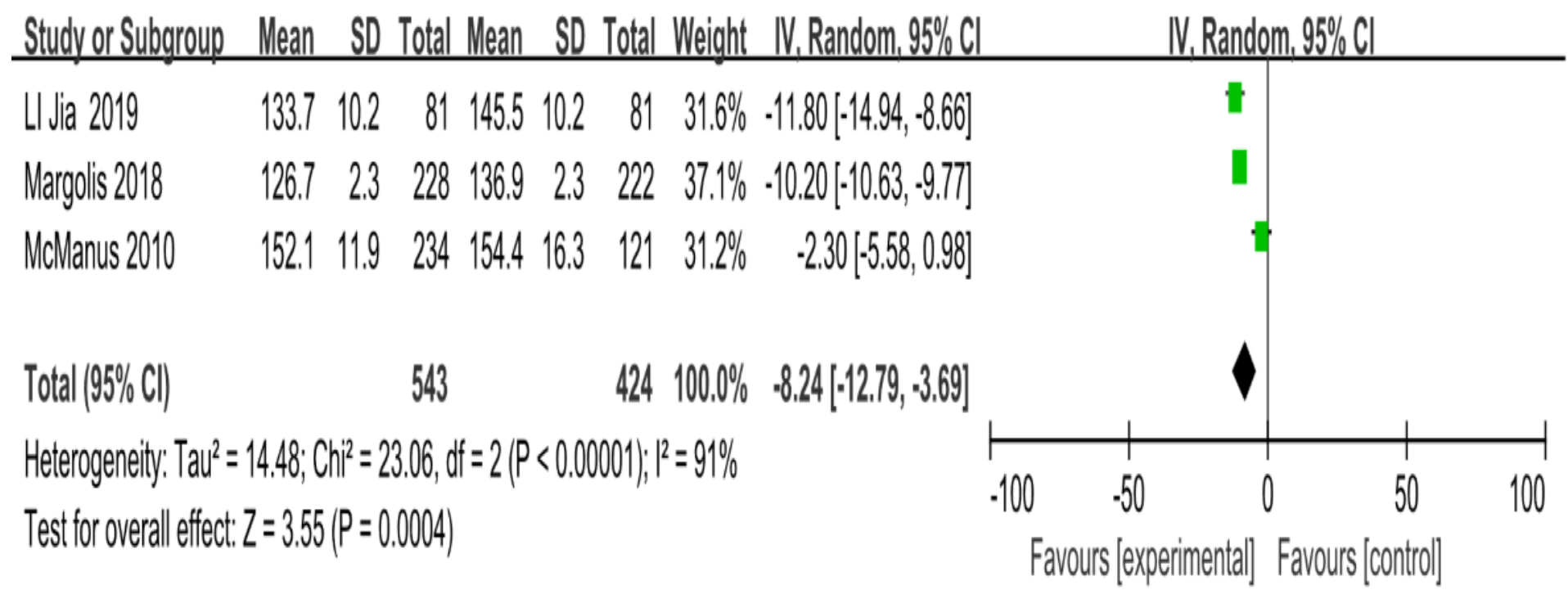

Figure 7

Forest plots of systolic blood pressure after 6 months of intervention between the intervention group and the control group Experimental Control Mean Difference Mean Difference Study or Subgroup Mean SD Total Mean SD Total Weight N, Random, 95\% Cl N, Random, 95\% C Cl LI Jia $2019 \quad 82.1 \quad 6.5 \quad 81 \quad 91.4 \quad 6 \quad 81 \quad 32.6 \% \quad-9.30[-11.23,-7.37]$ Margolis $2018 \quad 752.15228 \quad 81.72 .25 \quad 222 \quad 34.3 \%$ Mclanus 2010 $\quad 85 \quad 8.5 \quad 235 \quad 84.5 \quad 9.6 \quad 246 \quad 33.1 \%$ Total $(95 \%)$ C C $\quad 544 \quad 549100.0 \%$ Heterogeneity: $T_{a u}^{2}=16.96, \mathrm{Chi}^{2}=80.60$, df $=2(P<0.00001) ; 1^{2}=98 \%$ Test for overall effect: $z=2.14(P=0.03)$

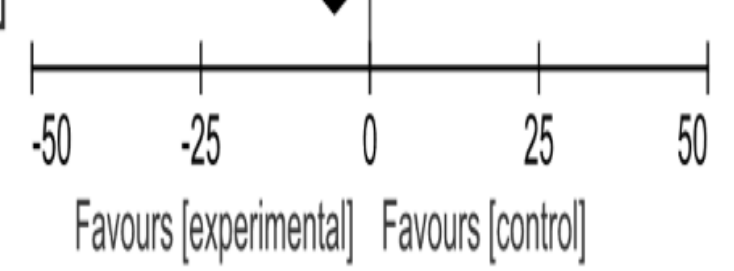

Figure 8

Forest plots of diastolic blood pressure after 6 months of intervention between the intervention group and the control group 